\title{
Image Encryption Scheme Based on Amplitude Modulation of Chaotic Signals
}

\author{
Chunlai Li $1,2,3^{*}$, Wenhua Hai $1,2^{*}$ \\ ${ }^{1}$ Department of Physics and Key Laboratory of Low-dimensional Quantum Structures and Quantum Control \\ of Ministry of Education, Hunan Normal University, Changsha, 410081, China. \\ 2 Synergetic Innovation Center for Quantum Effects and Applications, Hunan Normal University, Changsha, \\ 410081, China. \\ ${ }^{3}$ College of Physics and Electronics, Hunan Institute of Science and Technology, Yueyang, 414006, China. \\ * Corresponding author. Email: hnistlichl@163.com. \\ Manuscript submitted May 20, 2018; accepted June 8, 2018. \\ doi: 10.17706/jsw.13.8.421-436
}

\begin{abstract}
We introduce a three-dimensional chaotic system and reveal the relation between signal amplitude and the system parameters which can enrich the key for a cryptosystem. To study application of the relation to multi-media security, we propose an image encryption scheme based on the permutation-diffusion architecture. Security of immunity to known-plaintext attack and chosen-plaintext attack is ensured by adopting plaintext sequence and amplitude parameter to generate key stream with the ideology of one-time pad. Numerical experiments are implemented and prominent advantage of the theoretical scheme is confirmed.
\end{abstract}

Key words: Amplitude modulation, chaotic system, one-time pad, image encryption.

\section{Introduction}

With the rapid development of information technology and multimedia industry, digital image has been increasingly acquired, stored and transmitted. Consequently, the secure communication of confidential digital images over open channels has become vital and urgent requirement [1], [2]. Several traditional encryption schemes, such as DES (data encryption standard), AES (advanced encryption standard) and RSA (Rivest-Shamir-Adleman), are introduced for textual information based on confusion and diffusion principles [2]. In view of the theory by Shannon, practical cryptographic characteristics for image encryption include not only the bit confusion and diffusion, but also the computational unpredictability, and the sensitivity to keys and plain text [3], which can be commendably catered by the fundamental features of a chaotic system. Therefore, chaos can be thought of as a suitable candidate for image encryption [4-9].

In 1998, Fridrich introduced chaos-based image encryption with permutation-diffusion procedure at the first time [10]. Under this scheme, the position of image pixels is firstly changed to erase the high correlation between adjacent pixels; then the pixel values are modified sequentially using pseudorandom sequences, so that a tiny change in a pixel can spread out to as many pixels in the whole image as possible, and the correlations of adjacent pixels are broken simultaneously. Subsequently, the permutation-diffusion schemes and their extension, such as bit-level permutation approach [11], [12], plain-image confusion [13], enhanced key stream generator [1], [5], transform domain scheme are proposed [4], [14], [15]. Recently, 
some chaos-based image encryption algorithms with permutation-diffusion architecture are found to be insecure against different attacks, and have been subsequently broken [16]-[21]. The common weaknesses of these insecure schemes are summarized as below: (a) The algorithms for encryption/decryption are insensitive to the variations of the plain-image, and the key streams are independent of the plain-image, which favor known-plaintext attack and chosen-plaintext attack. (b) The chaotic sequences for permutation and diffusion are generated from different systems, and the procedures of permutation and diffusion are independent. Accordingly, the change of one of secret keys will only affect the permutation module or the diffusion procedure. (c) The schemes do not conform to the idea of one-time pad (OTP) since the key is unchanged in the procedure of encryption, which can't ensure perfect security.

In cryptography, OTP is the only known unbreakable cipher, and was proven mathematically to be perfectly secure by Shannon [22]. For OTP scheme, the private key is required to not only be perfectly random, but also be used only once, thus it must be as long as the message with an executed difficulty. In spite of the practical shortcomings, OTP continues to be used in DNA cryptography [23], quantum cryptography [24] and even in classical cryptography [25], when high security is desired. The amplitude parameter of a chaotic system is defined as that enabling modulating signal amplitude. If the amplitude parameter of a chaotic system is regarded as encryption key and changes with encryption process for every pixel, it can provide the private key as long as the message and result in possible realization of OTP.

In this paper, we present a practicable image encryption scheme for both combining permutation and diffusion and addressing the existed problems (weaknesses) mentioned above. Firstly, we propose a three-dimensional chaotic system with five linear terms and two quadratic product terms. Basic dynamical properties including the amplitude modulation are analyzed carefully. And the chaotic sequences are modified to obtain a chaotic key stream suiting for diffusion. Then the idea of OTP realized by correlating the parameter secret key and plain-image, is employed to shuffle the pixels. Security analyses and experiment results show the proposed scheme has high key sensitivity, high plaintext sensitivity and resistance to different attacks.

\section{The Chaotic System}

\subsection{System Description}

The introduced three-dimensional chaotic system holds five linear terms and two quadratic terms, as below

$$
\left\{\begin{array}{l}
\dot{x}_{1}=-a x_{1}+b x_{2} \\
\dot{x}_{2}=c x_{1}+d x_{3}-e x_{1} x_{3} \\
\dot{x}_{3}=f x_{1}^{2}-g x_{3}
\end{array}\right.
$$

Here, $x_{1}, x_{2}, x_{3}$ are state variables and $a, b, c, d, e, f, g$ are positive parameters. Similar to the ordinary chaotic systems, system (1) exhibits complex dynamical behaviors but different properties of amplitude modulation by varying some parameter.

By considering the condition $\dot{x}_{1}=0, \dot{x}_{2}=0, \dot{x}_{3}=0$, we get three equilibrium points, depicted by

$$
E_{0}(0,0,0), \quad E_{1}\left(x_{+}, \frac{a}{b} x_{+}, \frac{f}{g} x_{+}{ }^{2}\right), E_{2}\left(x_{-}, \frac{a}{b} x_{-}, \frac{f}{g} x_{-}^{2}\right) \text { with } x_{ \pm}=\frac{d}{2 e} \pm \sqrt{\frac{d^{2}}{4 e^{2}}+\frac{g c}{e f}} .
$$

The corresponding characteristic equation can be deduced as

$$
\Phi(\lambda)=-\lambda^{3}-(a+g) \lambda^{2}+\left(-a g+b\left(c-e x_{3}\right)\right) \lambda+2 b f x_{1}\left(d-e x_{1}\right)+b g\left(c-e x_{3}\right)
$$


Throughout this manuscript, we consider the parameter set

$$
S=\{a, b, c, d, e, f, g\}=\{10,6,28,1,1,0.1,5\}
$$

Unless explicitly stated otherwise. Thus the nonzero equilibrium points read $E_{1}(37.9199,63.1999,28.7584), E_{2}(-36.9199,-61.5332,27.2616)$. The characteristic roots corresponding to the equilibrium points are

$$
\begin{aligned}
& E_{0}: \lambda_{1}=-28, \lambda_{2}=20, \lambda_{3}=-3 . \\
& E_{1}: \lambda_{1}=-17.4608, \lambda_{2}=1.2304+9.7982 i, \lambda_{3}=1.2304-9.7982 i . \\
& E_{2}: \lambda_{1}=-17.7118, \lambda_{2}=1.3559+9.5793 i, \lambda_{3}=1.3559-9.5793 i .
\end{aligned}
$$

For the equilibrium points $E_{0}, \lambda_{1}$ and $\lambda_{3}$ are negative real number, $\lambda_{2}$ is positive real number. As a consequence, $E_{0}$ is a saddle-node of index 1 . But for the equilibrium points $E_{1}$ and $E_{2}, \lambda_{1}$ is a negative real number, $\lambda_{2}$ and $\lambda_{3}$ become a pair of complex conjugate roots with positive real parts, meaning saddle-focus points of index 2. The Lyapunov exponents of system (1) are calculated as $0.869,0.0,-22.51012$ for the parameter set $S$, and the corresponding Kaplan-Yorke dimension is 2.0386, denoting a fractional feature. Therefore, system (1) is chaotic, as depicted in Fig.1.

It deserves to be mentioned that system (1) holds two asymmetrical nonzero equilibrium points which is different from those of the similar systems [26-30], therefore, they are not topological equivalent, though all have similar attractors.
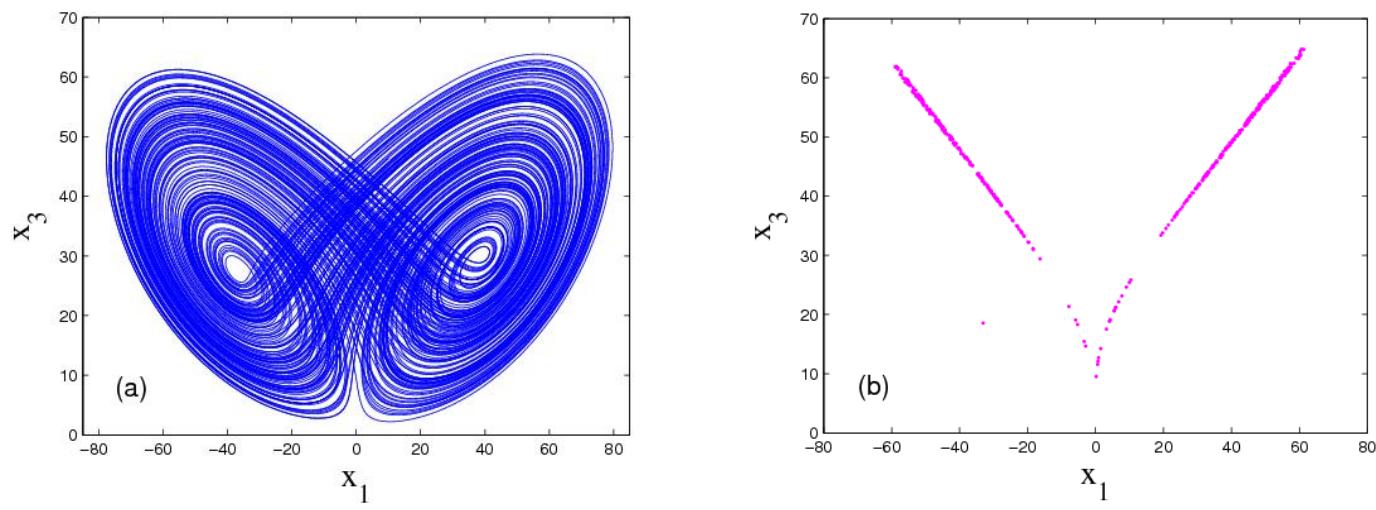

Fig. 1. $x_{1}-x_{3}$ phase portrait (a) and poincaré map (b) with $x_{2}=0$ and the parameter set $S=\{a, b, c, d, e, f, g\}=\{10,6,28,1,1,0.1,5\}$.

\subsection{Phase Modulation of Chaotic Signals}

In system (1), coefficient $d$ can control the phase of signals $x_{1}$ and $x_{2}$ simultaneously, which can be seen from the invariance with the transformation

$$
\left(x_{1}, x_{2}, x_{3}, a, b, c, d, e, f, g\right) \rightarrow\left(-x_{1},-x_{2}, x_{3}, a, b, c,-d, e, f, g\right) \text {. }
$$

The bifurcation diagrams for variables $x_{1}$ and $x_{2}$ are reverse symmetrical about $d=0$, which further confirms that the sign variation of $d$ can change the polarity of $x_{1}$ and $x_{2}$, regardless of the dynamics behavior, as described in Fig. 2.

\subsection{Amplitude Modulation of Chaotic Signals}


It is known from Refs. [31]-[33] that, for the quadratic chaotic systems, the coefficients of quadratic terms can control the signal amplitude partially or totally. Here we define such coefficients of a chaotic system as amplitude parameters. We will use the amplitude parameters to study the amplitude modulation of Eq. (1) for both the cases $f \neq e$ and $f=e$ as follows.
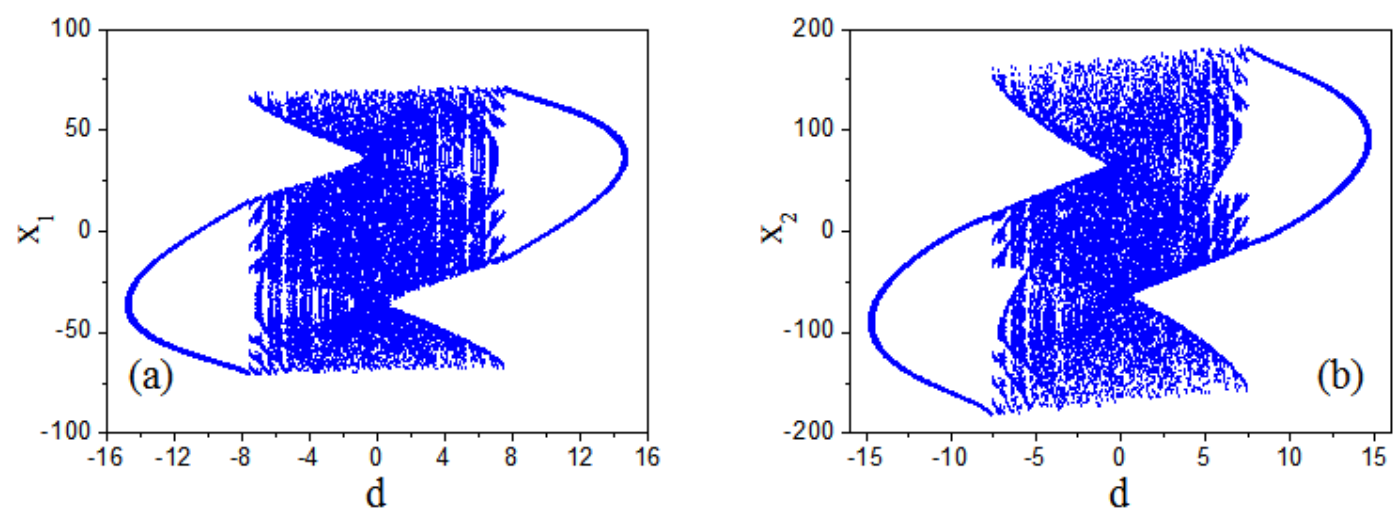

Fig. 2. Bifurcation diagram versus $d$ with the parameter set $S$ except for $d$.

Case $f \neq e$. The bifurcation diagram of system (1) with $e \in[0,10]$ is depicted in Fig.3 (a). Superficially, the coefficient $e$ can control the amplitude of signal $x_{1}$ nonlinearly. But one can see that there emerges visible period doubling bifurcation or periodic window from the enlarged view, as shown in Fig.3 (b). The bifurcation diagram versus coefficient $f$ further demonstrates that not all coefficients of quadratic terms can modulate the signal amplitude, seen in Fig.3 (c).
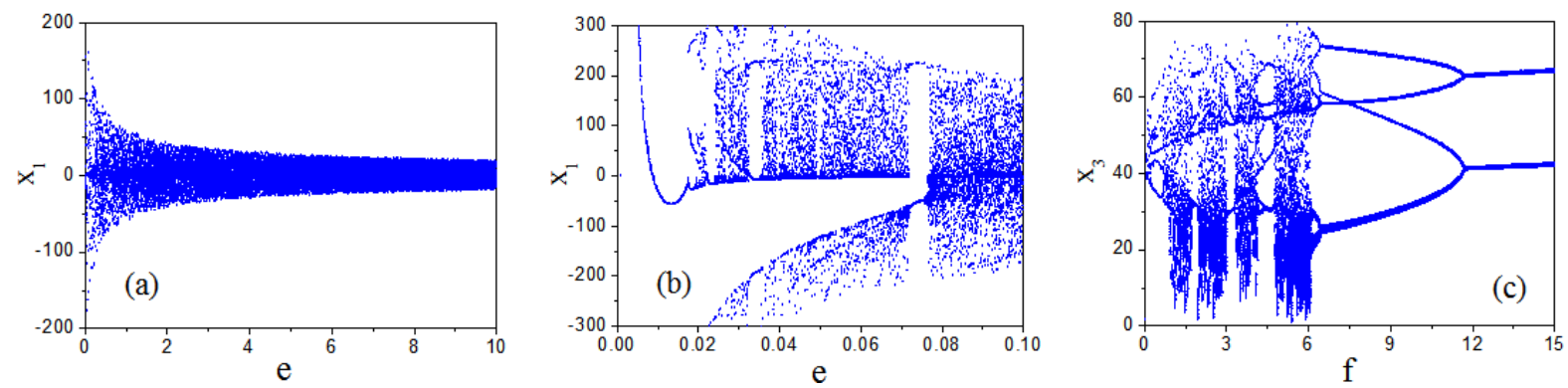

Fig. 3. (a), (b) Bifurcation diagram versus $e$ with the parameter set $S$ except for $e$;

(c) Bifurcation diagram versus $f$ with the parameter set $S$ except for $f$.

Spontaneously, when considering the property of amplitude modulation for chaotic system, we raise a puzzled yet worth to be explored question "what's the necessary condition for possibly modulating signal amplitude of chaotic system?" It's known that the equilibrium point of dynamical system denotes the equilibrium position of unstable motion with zero velocity, and the nonzero equilibrium points will accordingly deviate from the original position when rescaling the trajectory of chaotic attractor. Therefore, for the chaotic system with multiple equilibrium points, the possible prerequisite for amplitude modulation of chaotic signals can be summarized as: (a) in the mathematical expression of nonzero equilibrium point, the amplitude parameter is symmetrical about some axis; (b) and the amplitude parameter can modulate the location of the nonzero equilibrium point in coordinate plane. 
Case $f=e$. Considering the expression of nonzero equilibrium point of system (1) for the case $f \neq e$, any one of parameters $e$ and $f$ of nonlinear term can't modulate the location of equilibrium point $E_{1}$ or $E_{2}$. Consequently, parameter $e$ or $f$ can't execute amplitude modulation. Now we introduce a unified parameter $e$ for nonlinear term $x_{1} x_{3}$ and $x_{1}{ }^{2}$, resulting in the new case $f=e$ of Eq. (1) and the nonzero equilibrium points

$$
\begin{gathered}
E_{1}\left(\frac{1}{e}\left(\frac{d}{2}+\sqrt{\frac{d^{2}}{4}+g c}\right), \frac{1}{e}\left(\frac{a d}{2 b}+\frac{a}{b} \sqrt{\frac{d^{2}}{4}+g c}\right), \frac{1}{e}\left(\frac{d^{2}}{2 g}+c+\frac{d}{2 g} \sqrt{\frac{d^{2}}{4}+g c}\right)\right), \\
E_{2}\left(\frac{1}{e}\left(\frac{d}{2}-\sqrt{\frac{d^{2}}{4}+g c}\right), \frac{1}{e}\left(\frac{a d}{2 b}-\frac{a}{b} \sqrt{\frac{d^{2}}{4}+g c}\right), \frac{1}{e}\left(\frac{d^{2}}{2 g}+c-\frac{d}{2 g} \sqrt{\frac{d^{2}}{4}+g c}\right)\right) .
\end{gathered}
$$

The set of symmetrical axis for points $E_{1}$ and $E_{2}$ is $\left(\frac{d}{2 e}, \frac{a d}{2 b e}, \frac{d^{2}}{2 e g}+\frac{c}{e}\right)$. In addition, the unified parameter $e$ can modulate the location of the nonzero equilibrium points according to $\frac{1}{e}$ respectively. As a result, the parameter $e$ can execute amplitude modulation for signal $x_{1}, x_{2}, x_{3}$ according to $\frac{1}{e}$. The corresponding signal amplitude and Lyapunov exponent spectrum versus $e$ are depicted in Fig.4.
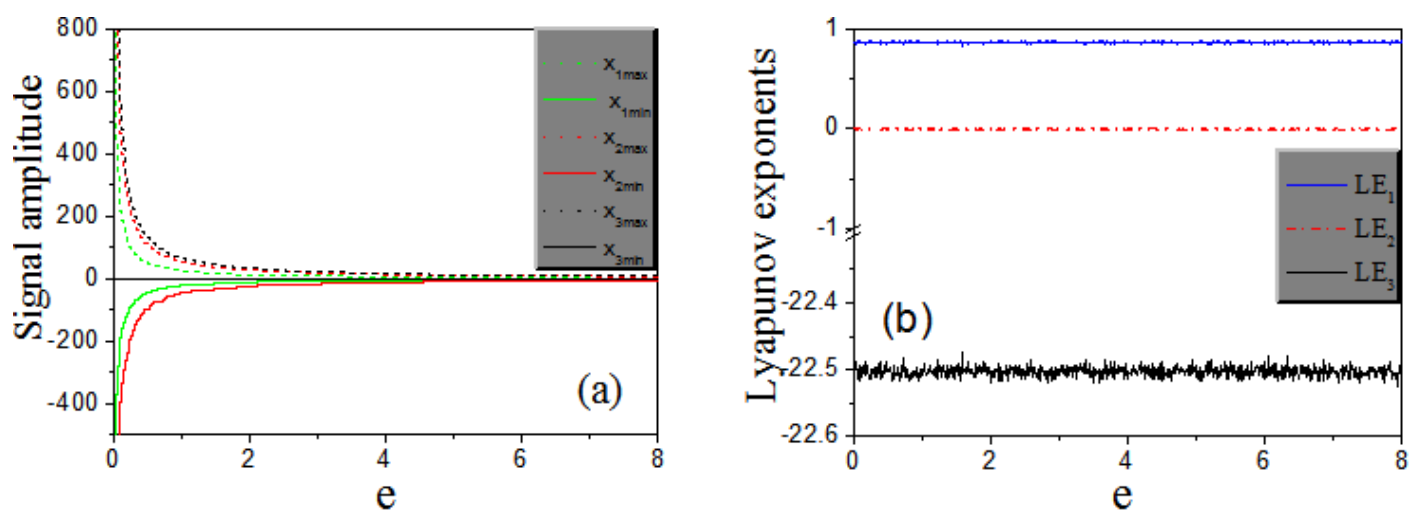

Fig. 4. Signal amplitude (a) and Lyapunov exponent spectrum (b) versus $e$ with the parameter set $S$ except for $e$ and $f$.

\section{Image Encryption Scheme Based on Amplitude Modulation of Chaotic Signals}

\subsection{Improved Chaotic Sequences}

The Logistic map is a simple chaotic system with one-dimensional dynamical equation yet complex behavior, expressed as

$$
x(n+1)=\alpha x(n)(1-x(n))
$$

When the parameter is selected as $\alpha \in(3.9,4]$, the system (4) is chaotic and the sequence $x(n)$ is between 0 and $1[5,7]$. 
Based on Golomb's theory [1], three properties should hold for an ideal pseudo-random sequence: (a) the AC (autocorrelation) is the delta function; (b) the CC (cross correlation) is zero; (c) uniform distribution. Experimental results show that the sequences $x_{1}, x_{2}, x_{3}$ generated by new system (1) with $f=e$ and the sequence $x$ generated by system (4) are not the ideal pseudo-random sequences, which need to be modified, see Figs.5 (a), (c) and Figs.6 (a). In the encryption scheme, we make the pretreatment for these sequences, as below:

$$
\begin{aligned}
& h_{1}=x_{1} \times 10^{2}-\operatorname{round}\left(x_{1} \times 10^{2}\right) \\
& h_{2}=x_{2} \times 10^{2}-\operatorname{round}\left(x_{2} \times 10^{2}\right) \\
& h_{3}=x_{3} \times 10^{2}-\operatorname{round}\left(x_{3} \times 10^{2}\right) \\
& h_{4}=x \times 10^{2}-\operatorname{round}\left(x \times 10^{2}\right)
\end{aligned}
$$

In (5), round $\left({ }^{*}\right)$ stands for rounding $*$ to the nearest integer. As shown in Fig.5 (b), (c), the autocorrelations of sequences $h_{1}$ to $h_{4}$ verge on the ideal delta function. And the cross correlation between sequences $h_{1}$ and $h_{2}$ is close to zero, depicted as Fig.6 (b). Further, we know that the modified sequences hold uniform distribution between -0.5 and 0.5 . Therefore, the modified sequences in (5) have better performance for image encryption.
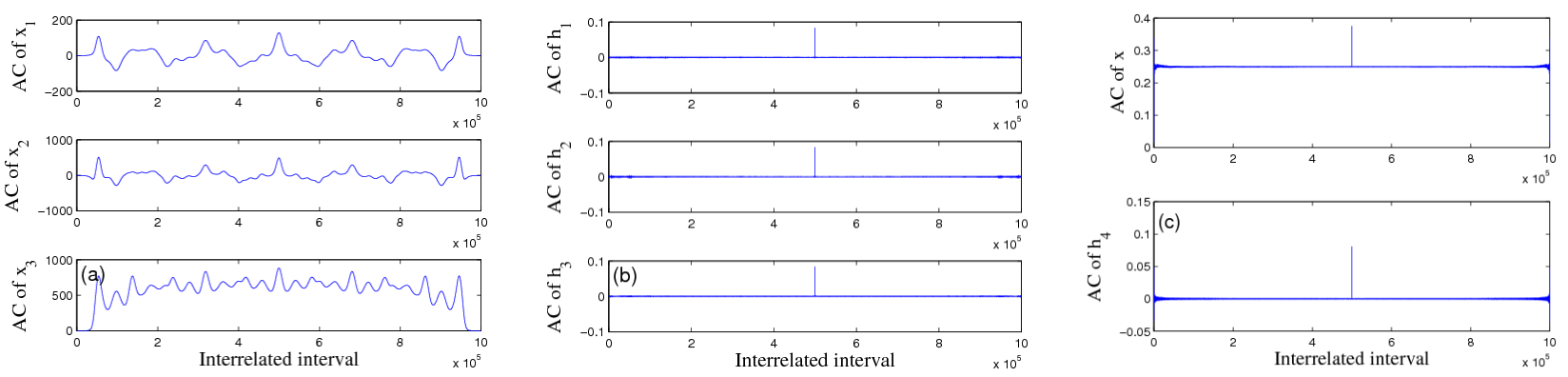

Fig. 5. Autocorrelation series for the pseudo-random sequences and the modified forms.
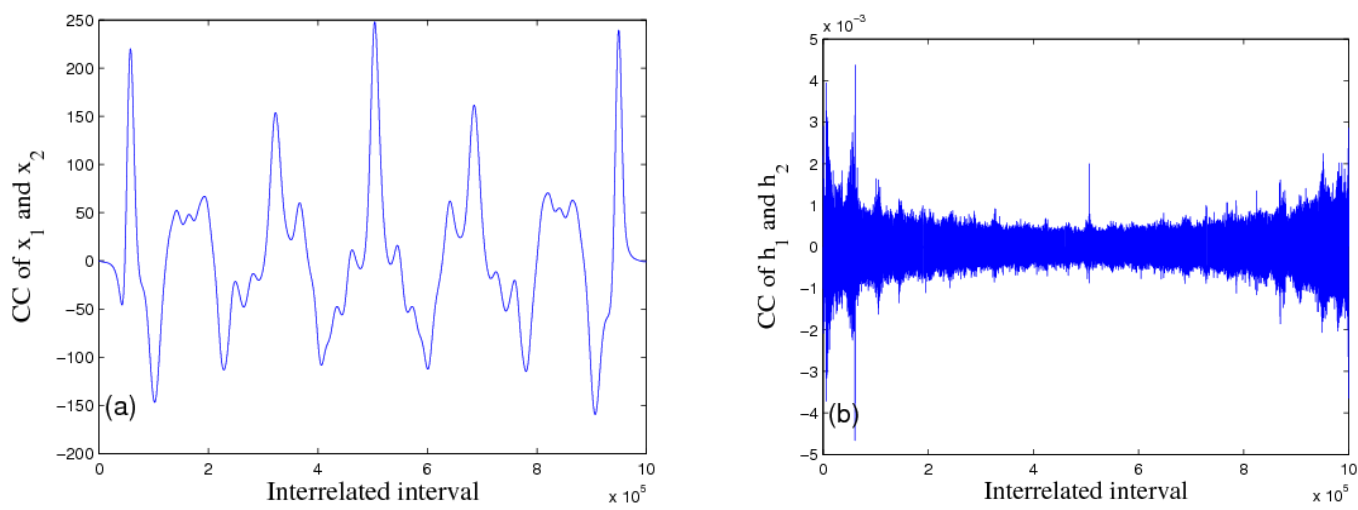

Fig. 6. Cross correlation series for $x_{1}, x_{2}$ and $h_{1}, h_{2}$.

\subsection{Image Encryption Scheme}

The block diagram of the suggested image encryption scheme is shown in Fig.7. This scheme can ensure high security for holding the following features: (a) The chaotic sequences for permutation and diffusion are simultaneously generated from Logistic map and new chaotic system, thus the change of one of secret keys will affect both the permutation module and the diffusion procedure. (b) The 
plaintext sequence and amplitude parameter are adopted to generate key stream based on the idea of OTP, which can effectively fend off known-plaintext attack and chosen-plaintext attack. (c) The chaotic key stream is modified for diffusion process based on the properties of ideal pseudo-random sequence. (d) There exists a big key space to ensure a high security level. The encryption procedure is described as follows.

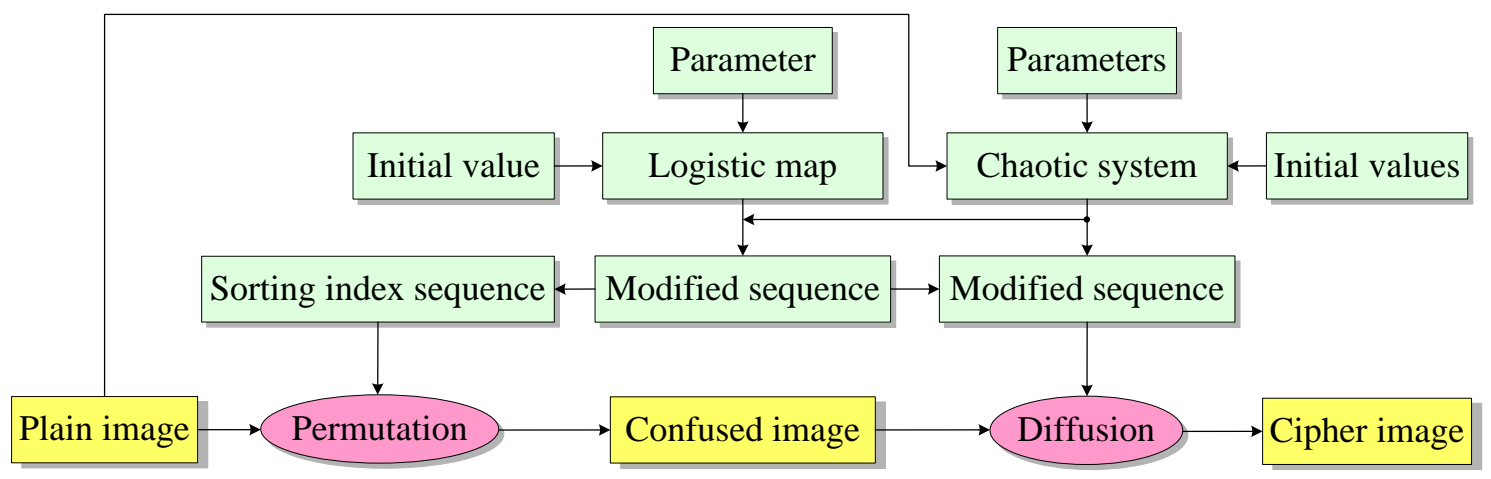

Fig. 7. Block diagram of the suggested image encryption scheme.

Step 1. Input the original image $I$ with size $L=M \times N$. The $L$ pixels are arranged as a one-dimensional array from the upper left to the lower right, which is represented by $P=\left(P_{0}, P_{1}, \ldots, P_{\mathrm{L}-1}\right)$.

Step 2. Generate chaotic sequence $x=\left(x_{1}, x_{2}, \ldots, x_{\mathrm{L}}\right)$ by Logistic map (4) with parameter $\alpha \in(3.9,4]$ and initial value $x_{0}$.

Step 3. Generate chaotic sequences $x_{1}=\left(x_{11}, x_{12}, \ldots, x_{1 \mathrm{~L}}\right), x_{2}=\left(x_{21}, x_{22}, \ldots, x_{2 \mathrm{~L}}\right)$ and $x_{3}=\left(x_{31}, x_{32}, \ldots, x_{3 \mathrm{~L}}\right)$ by the modified chaotic system (6) with parameter set $(a, b, c, d, e, g)$ and initial condition $\left(x_{10}, x_{20}, x_{30}\right)$.

$$
\left\{\begin{array}{l}
\dot{x}_{1}=-a x_{1}+b x_{2} \\
\dot{x}_{2}=c x_{1}+d x_{3}-(e+k) x_{1} x_{3} \\
\dot{x}_{3}=(e+k) x_{1}^{2}-g x_{3}
\end{array}\right.
$$

with $k=\left(k_{1}, k_{2}, \cdots, k_{L}\right), \quad k_{i}=\bmod \left(\frac{P_{i}}{256 \pi} \times 10^{8}, 256\right) / 256$.

Step 4. Generate chaotic sequences $y_{1}=\left(y_{11}, y_{12}, \ldots, y_{1 \mathrm{~L}}\right), y_{2}=\left(y_{21}, y_{22}, \ldots, y_{2 \mathrm{~L}}\right)$ by

$$
\begin{aligned}
& y_{1}=\tanh \left(e^{x}+x+x_{1}\right) \\
& y_{2}=\bmod \left(\left(y_{1}-a b s\left(y_{1}\right)\right) \times 10^{15}, 256\right)
\end{aligned}
$$

Step 5. Sort sequence $y_{2}$ by ascending order to get the corresponding index sequence. According to the index sequence, number the one-dimensional pixels $P$ and adjust pixel positions sequentially. Rearrange the scrambled pixels from left to right and top to bottom to get the confused image $I_{\mathrm{c}}$.

Step 6. The chaotic sequences of system (6) are modified according to Golomb's theory, and obtain $h_{1}, h_{2}, h_{3}$.

Step 7. Generate one-dimensional chaotic key stream $S$ with size $L$ for diffusion process by the formulas

$$
H=\bmod \left(\left(h_{1}+h_{2}-a b s\left(h_{1}+h_{2}\right)\right) \times 10^{14}+\left(h_{3}-a b s\left(h_{3}\right)\right) \times 10^{15}+\left(h_{4}-a b s\left(h_{4}\right)\right) \times 10^{16}, 256\right)
$$




$$
S=\bmod \left(\text { floor }(H) \times 2^{16}, 256\right)
$$

Step 8 . Modify the pixel values of the confused image by performing forward diffusion and reverse diffusion, as below

$$
\begin{aligned}
& I_{d}(i)=I_{d}(i-1) S(i) \oplus I_{c}(i) \\
& I_{d}(i)=I_{d}(i+1) S(i) \oplus I_{c}(i)
\end{aligned}
$$

The pixel values of image $I_{\mathrm{c}}$ are completely diffused by two round diffusions with the key stream $S$, thus we get the ciphered image $I_{\mathrm{d}}$ via the exclusive OR operation.

The decryption procedure is an inverse process of encryption procedure.

\section{Experimental Results and Security Analysis}

In this section, by adopting different standard images with 8 -bit grayscale and size of $512 \times 512$, a series of numerical experiments have been conducted to check the security of the introduced cryptosystem. The initial value and parameter of Logistic map are chosen as $\alpha=4, x_{0}=0.02$. The initial conditions and parameters of system (6) are fixed as $x_{10}=0.02, x_{20}=0.1, x_{30}=0.03, a=10, b=6, c=28, d=1$, $e=1, g=5$.

The experimental results for Lena image are shown in Fig.8. And several tests will be discussed to analyze the security level of the proposed encryption algorithm in the following subsections.

\subsection{Key Space Analysis}

Kerckhoff pointed out that the information system should be of security even if the system is publicly available except the key [34]. Therefore, a good encryption scheme should provide a large enough key space against any brute force attacks. In this encryption technique, the four initial values $x_{0}, x_{10}, x_{20}, x_{30}$ and seven parameter values $\alpha, a, b, c, d, e, g$ for systems' setup can be viewed as secret keys. Accordingly, if the computational precision is $10^{-15}$, the total key space size is bigger than $10^{15 \times 11}=10^{165}$, which is large enough to ensure a high security against brute-force attacks.

\subsection{Sensitivity Analysis}

A good encryption algorithm should be sensitive to the mismatches with respect to the original images or any secret keys on the whole cipher-image, for this reason, two common types of sensitivity analysis are executed in the following experiments.

\subsubsection{Key sensitivity}

The key sensitivity (KS) can be measured by calculating the Number of Pixel Change Rate (NPCR) and Unified Average Changing Intensity (UACI), as the following formulas

$$
\begin{gathered}
N P C R=\frac{1}{L} \sum_{i=1}^{M} \sum_{j=1}^{N} \operatorname{Dif}(i, j) \times 100 \% \\
U A C I=\frac{1}{L\left(2^{Q}-1\right)} \sum_{i=1}^{M} \sum_{j=1}^{N}\left|I_{d 1}(i, j)-I_{d 2}(i, j)\right| \times 100 \%
\end{gathered}
$$


where $L=M \times N$ is the size of the cipher-image; $Q$ is the number of bits, for images holding allocations of 8 bits/pixel of gray-scale, $2^{Q}-1=255 ; I_{\mathrm{d} 1}$ and $I_{\mathrm{d} 2}$ are two cipher-images corresponding to the right and mismatched keys; Diff $(i, j)$ denotes the difference between $I_{\mathrm{d} 1}$ and $I_{\mathrm{d} 2}$.
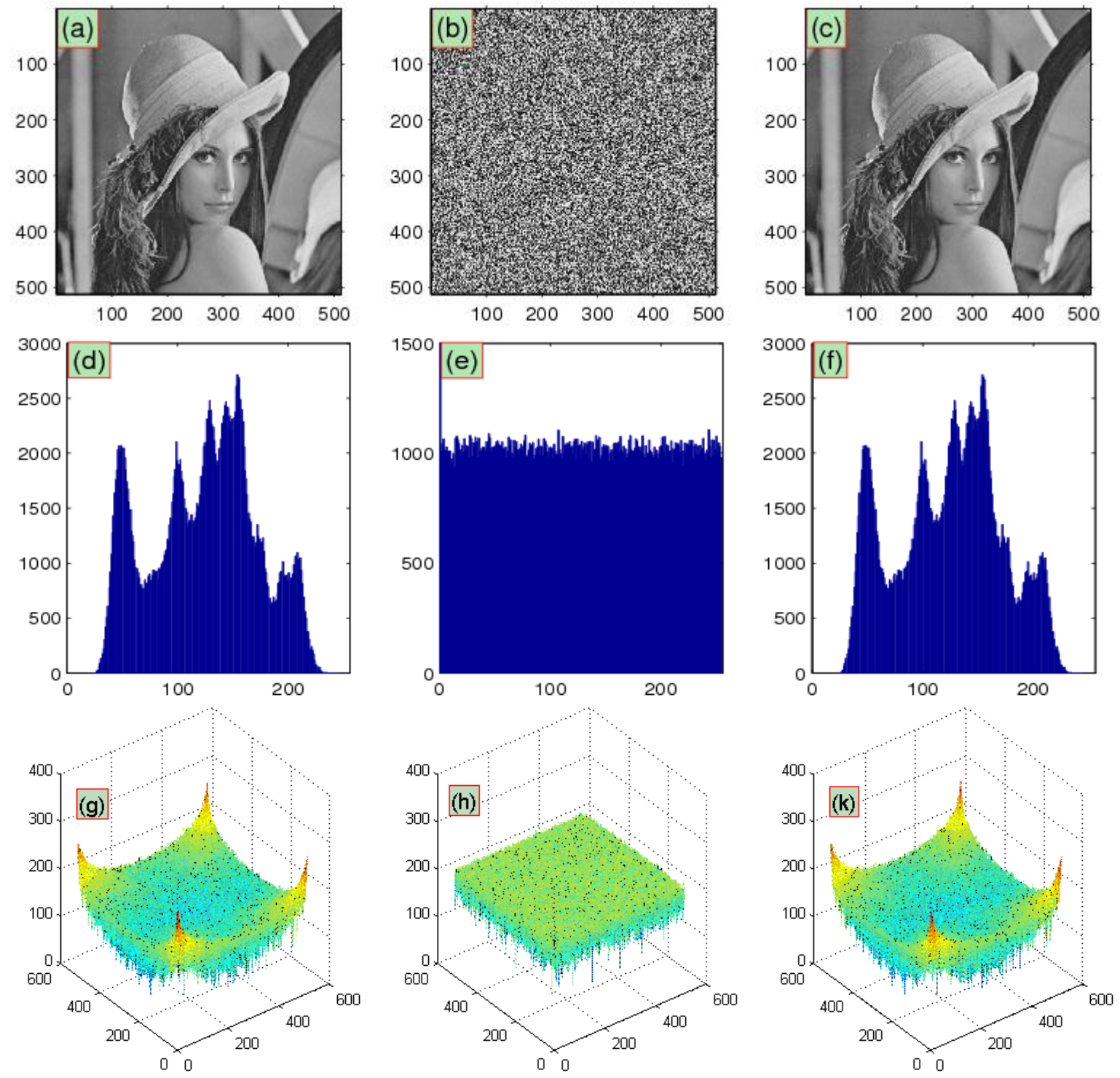

Fig. 8. (a) original Lena image; (b) Encrypted image; (c) Decrypted image; (d) Histogram of Lena image; (e) Histogram of the encrypted image; (f) Histogram of the decrypted image; (g) Amplitude spectrum of Lena image; (h) Amplitude spectrum of encrypted image; (k) Amplitude spectrum of decrypted image.

We first encrypt the standard images Lena, Scenery and Girla, using the right keys $\alpha=4, x_{0}=0.02$, $x_{10}=0.02, x_{20}=0.1, x_{30}=0.03, a=10, b=6, c=28, d=1, e=1, g=5$. Then we encrypt the same images with tiny change of keys. For all cryptographic systems, the idea results of NPCR and UACI are $100 \%$ and $33.33 \%$, respectively. Therefore, experimental results of the proposed encryption are close to the expected estimate, as listed in Table 1 . Thus, a high sensitivity of key is provided by the proposed scheme. 
Table 1. Key Sensitivity for Different Standard Images

\begin{tabular}{|c|c|c|c|c|c|c|c|c|c|c|c|c|c|c|c|}
\hline \multicolumn{10}{|c|}{$\begin{array}{c}\text { Sogistic } \\
\text { Map }\end{array}$} & \multicolumn{10}{|c|}{ NPCR/\% } & \multicolumn{3}{c|}{ UACI/\% } \\
\hline$\Delta \alpha$ & $\Delta x_{0}$ & $\Delta a$ & $\Delta b$ & $\Delta c$ & $\Delta g$ & $\Delta e$ & $\Delta x_{10}$ & $\Delta x_{20}$ & $\Delta x_{30}$ & Lena & Scenery & Girla & Lena & Scenery & Girla \\
\hline $\begin{array}{c}10^{-} \\
15\end{array}$ & 0 & 0 & 0 & 0 & 0 & 0 & 0 & 0 & 0 & 99.6029 & 99.6071 & 99.6136 & 33.2705 & 33.5786 & 33.5705 \\
\hline 0 & $10^{-}$ & 0 & 0 & 0 & 0 & 0 & 0 & 0 & 0 & 99.6235 & 99.5941 & 99.5964 & 33.4416 & 33.4426 & 33.5246 \\
\hline 0 & 0 & 0.01 & 0 & 0 & 0 & 0 & 0 & 0 & 0 & 99.6254 & 99.6014 & 99.6178 & 33.2859 & 33.4857 & 33.5330 \\
\hline 0 & 0 & 0 & $10^{-}$ & 0 & 0 & 0 & 0 & 0 & 0 & 99.6067 & 99.6048 & 99.5941 & 33.4125 & 33.5360 & 33.5056 \\
\hline 0 & 0 & 0 & 0 & 0.01 & 0 & 0 & 0 & 0 & 0 & 99.6117 & 99.5880 & 99.5945 & 33.4434 & 33.3556 & 33.4177 \\
\hline 0 & 0 & 0 & 0 & 0 & $10^{-}$ & 0 & 0 & 0 & 0 & 99.6109 & 99.6082 & 99.5861 & 33.4224 & 33.6465 & 33.4880 \\
\hline 0 & 0 & 0 & 0 & 0 & 0 & $10^{-}$ & 0 & 0 & 0 & 99.6143 & 99.6456 & 99.5945 & 33.2362 & 33.4934 & 33.4980 \\
\hline 0 & 0 & 0 & 0 & 0 & 0 & 0 & $10^{-}$ & 0 & 0 & 99.6315 & 99.6185 & 99.6067 & 33.5187 & 33.4595 & 33.6973 \\
\hline 0 & 0 & 0 & 0 & 0 & 0 & 0 & 0 & $10^{-}$ & & 99.5811 & 99.6075 & 99.6010 & 33.3366 & 33.3245 & 33.5061 \\
\hline 0 & 0 & 0 & 0 & 0 & 0 & 0 & 0 & 0 & $10^{-}$ & 99.6277 & 99.5998 & 99.6155 & 33.2998 & 33.5342 & 33.6493 \\
\hline
\end{tabular}

\subsubsection{Plaintext sensitivity}

The encryption technique can effectively resist differential attack if that a slight change in the plain-image makes the cipher-image vary greatly, which can also be tested by calculating the values of NPCR and UACI. For the plaintext sensitivity (PS), $I_{\mathrm{d} 1}$ and $I_{\mathrm{d} 2}$ are respectively the cipher-image before and after the change of one pixel of the plain image. The experimental results for the standard images Lena, Scenery and Girla are depicted in Table 2. It is clear that the values of NPCR and UACI remain in the vicinity of the expected values, thus the proposed scheme is extreme sensitive with respect to the little change of plain-image and can effectively resist differential attack.

Table 2. Plaintext Sensitivity for Different Standard Images

\begin{tabular}{|c|c|c|c|c|c|}
\hline \multicolumn{3}{|c|}{ NPCR/\% } & \multicolumn{3}{c|}{ UACI/\% } \\
\hline Lena & Scenery & Girla & Lena & Scenery & Girla \\
\hline 99.6174 & 99.6044 & 99.6361 & 33.4027 & 33.5370 & 33.5818 \\
\hline
\end{tabular}

\subsection{Statistical Analysis}

According to Shannon's theory, the encryption process should prevent the cipher-image from suffering any statistical attack [3], [22]. There is strong correlation among adjacent pixels for plain images. Statistical analysis is mainly to observe the confusion and diffusion properties of the encrypted image and the difference with the original image. In order to prove the security of the proposed cryptographic scheme, several statistical tests such as histogram, spectrum, information entropy, correlation are performed in this subsection.

\subsubsection{Histograms of image}


The histogram reveals the statistical characteristics of image and the distribution information of pixel values. Histograms of the original Lena image and its encrypted and decrypted images are shown in Fig. 8 (d-f). It's known that the histogram of encrypted image distributes nearly uniform and completely differ from the histograms of the original and decrypted images. Hence, the histogram of encrypted image does not reveal any statistical information of the original image. Thus, it is difficult for attackers to recover the plain image from statistical feature of ciphered image.

\subsubsection{Spectrums of image}

Similar to the histogram of image, the spectrum also reflects the distribution of the corresponding image in frequency domain. The distributions of the amplitude spectrum for the original Lena image and the corresponding encrypted and decrypted images are shown in Fig. 8 (g-k). It can be seen that there exists ridges and valleys in Fig. 8 (g) and Fig. 8 (k), but the amplitude spectrum of the encrypted image in Fig. 8 (h) is fairly uniform in the whole frequency distribution. What's more, the amplitude spectrum of the decrypted image is almost equivalent to the original one. Therefore, the proposed technique is strong enough to resist any statistical attack.

\subsubsection{Information entropy}

Information entropy is an important measure of the uncertainty of randomness. Let $p\left(s_{\mathrm{i}}\right)$ be the probability of information source $s_{\mathrm{i}}$, and $N$ is the number of bits for each information symbol $s_{\mathrm{i}}$. The information entropy can be calculated as

$$
H(s)=-\sum_{i=1}^{2^{N}-1} p\left(s_{i}\right) \log _{2} p\left(s_{i}\right)
$$

The entropy of experimental result for the standard images Lena, Scenery and Girla are 7.9972, 7.9973, 7.9973, respectively. Compared with the algorithms from Refs. [1, 4], the obtained result for the cipher-image Lena is more close to the ideal value 8 . Therefore, the probability of information leakage of the proposed scheme is very little and the ciphered image is secure against any kind of entropy attack.

\subsubsection{Correlations of two adjacent pixels}
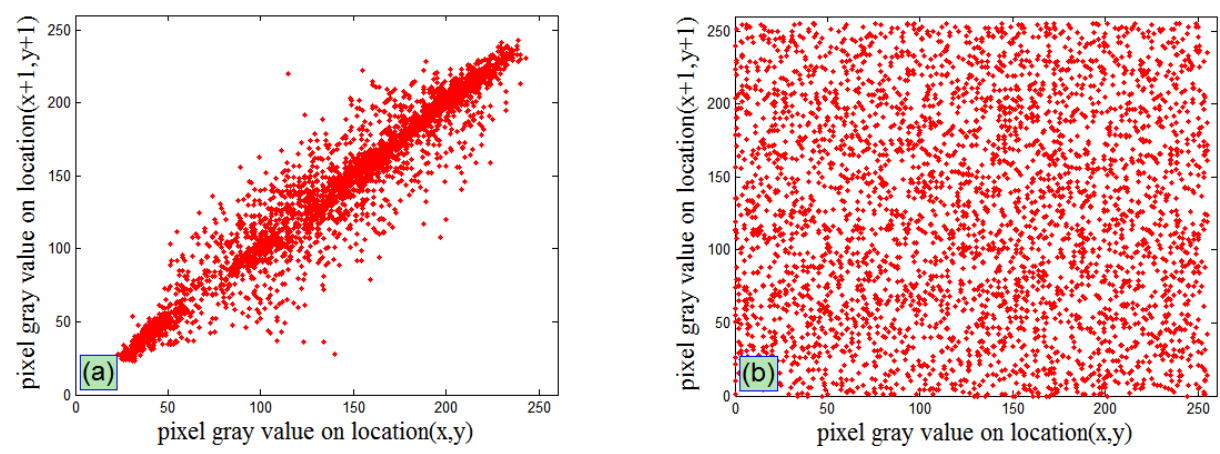

Fig. 9. (a) Correlations in the plain-image Lena; (b) Correlations in the cipher-image Lena.

A secure encryption scheme should reduce the correlation between adjacent image pixels to withstand statistical attack. Thus, we randomly select 10,000 pairs of two adjacent pixels in horizontal, 
vertical and diagonal directions from the original image and the cipher-image to test the correlation. The correlation coefficient $R_{x y}$ will be calculated by using the following equation

$$
R_{x y}=\operatorname{cov}(x, y) / \sqrt{D(x) D(y)}
$$

with $\operatorname{cov}(x, y)=\frac{1}{N} \sum_{i=1}^{N}\left(x_{i}-E(x)\right)\left(y_{i}-E(y)\right), \quad D(x)=\frac{1}{N} \sum_{i=1}^{N}\left(x_{i}-E(x)\right)^{2}, \quad E(x)=\frac{1}{N} \sum_{i=1}^{N} x_{i}$, where $x$ and $y$ are gray-scale values of the adjacent pixels in the image.

Fig. 9 shows the correlation distribution of two diagonally adjacent pixels in the original Lena image and that in the ciphered image, with the corresponding correlation coefficients 0.9739 and 0.0014. The test results for different standard images are concluded in Table 3. From Table 3, it can be seen that the proposed scheme approximates zero correlation, revealing high-level security.

Table 3. Correlation Coefficients for the Standard Images Girla, Scenery and Lena

\begin{tabular}{|c|c|c|c|c|c|c|}
\hline \multirow{2}{*}{ Direction } & \multicolumn{3}{|c|}{ Plain-image } & \multicolumn{3}{c|}{ Cipher-image } \\
\cline { 2 - 7 } & Lena & Scenery & Girla & Lena & Scenery & Girla \\
\hline Horizontal & 0.9858 & 0.9690 & 0.9686 & -0.0090 & 0.0011 & -0.0035 \\
\hline Vertical & 0.9904 & 0.9832 & 0.9889 & -0.0019 & -0.0012 & 0.0036 \\
\hline Diagonal & 0.9739 & 0.9759 & 0.9544 & 0.0014 & 0.0012 & 0.00004 \\
\hline
\end{tabular}

\subsection{Noise Attack Analysis}
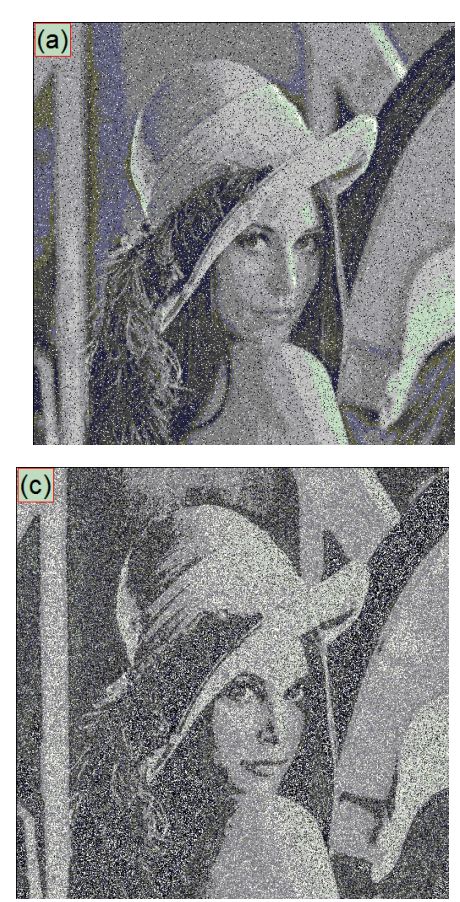
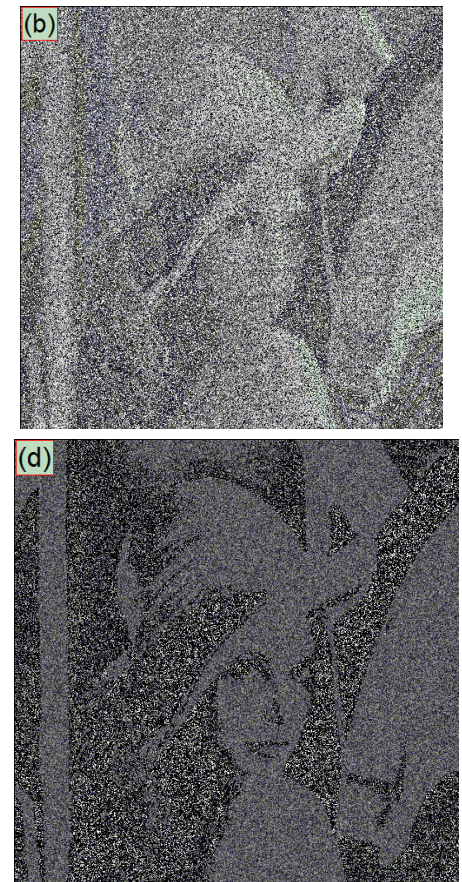

Fig. 10. Decrypted images disturbed by salt-and-pepper noise with (a) $R=0.1$ and (b) $R=0.4$; decrypted images disturbed by Gaussian noise with (c) $R=0.1$ and (d) $R=0.4$.

The image is often corrupted by different noises in processing and transmission. To analyze the immunity to different noise of this scheme, we add the salt-and-pepper noise and Gaussian noise to the ciphered image of Lena in the way: $I_{\mathrm{d}}^{\prime}(x, y)=I_{\mathrm{d}}(x, y)(1+R N(x, y))$, in which $I_{\mathrm{d}}(x, y)$ and $I_{\mathrm{d}}(x, y)$ denote the ciphertext and the noise-disturbed ciphertext respectively, $N(x, y)$ is the salt-and-pepper noise or 
Gaussian noise, $R$ denotes the noise strength. The decryption process is performed with correct keys except that the ciphertext is disturbed by different noises. Fig. 10 (a) and (b) show the results of decrypting ciphertext disturbed by salt-and-pepper noise when $R$ is set to 0.1 and 0.4 . Fig. 10 (c) and (d) show the results of decrypting ciphertext disturbed by Gaussian noise when $R$ is set to 0.1 and 0.4 . As we know that the decrypted plain images become more fuzzy with a larger strength $R$, but the main information of the plain images can be recognized. Therefore, the proposed encryption scheme can resist noise attack to some extent.

\subsection{Occlusion Attack Analysis}

The image is mostly also damaged by occlusion in processing and transmission. We perform the decryption process with all correct keys except that the encrypted image is occluded partly. Fig. 11 (a) displays the occluded ciphertext which is cut by $25 \%$ at the central and Fig. 11(b) depicts the recovered plain image. Fig. 11(c) displays the occluded ciphertext cut by $50 \%$ at the lower-left corner and top-right corner, and Fig. 11 (d) shows the decrypted image. From the recovered images, it's found that the content of the original image can be identified visually. Therefore, the introduced encryption scheme is proved to be immune to occlusion attack.
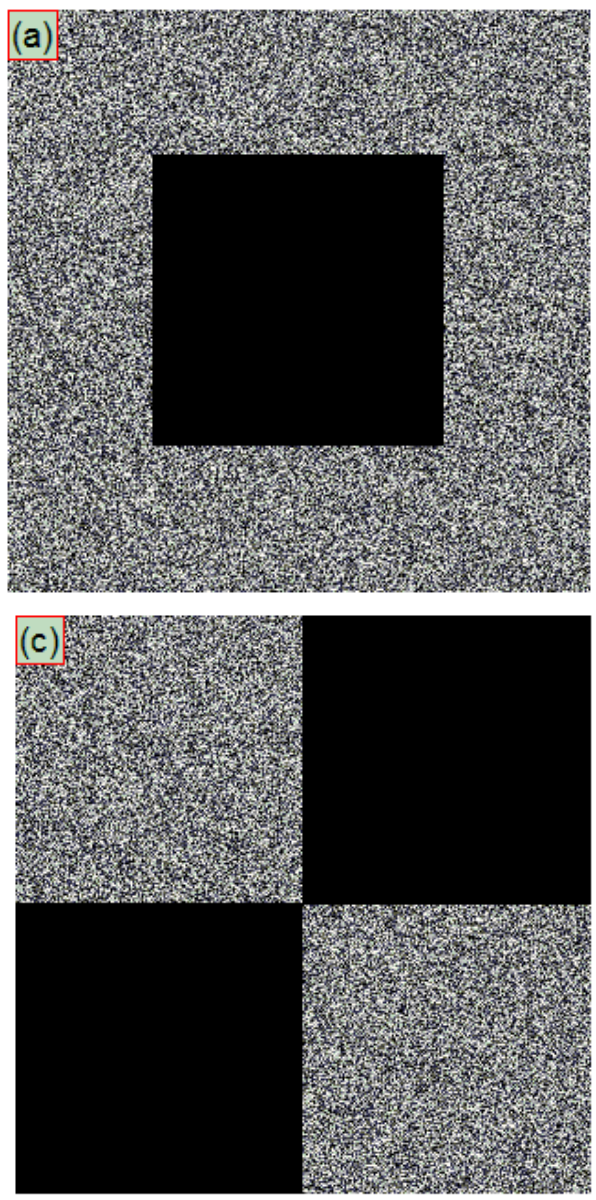
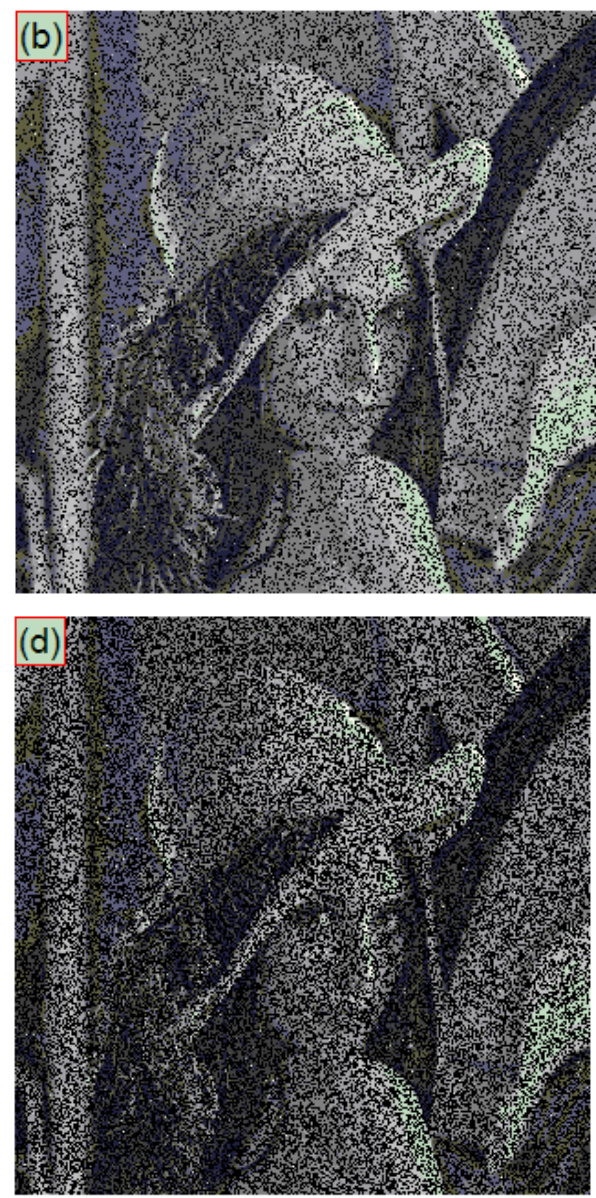

Fig. 11. (a) Ciphertext with 25\% occlusion and (b) the corresponding decrypted image; (c) ciphertext with $50 \%$ occlusion and (d) the corresponding decrypted image.

\section{Discussion and Conclusion}


A practical scheme for image encryption includes not only bit confusion and diffusion, but also computational unpredictability and sensitivity to keys and plain text, which can be commendably catered by chaotic systems. And the chaotic system with amplitude parameter can execute a possible realization for one-time pad since it can provide enough key than the message. In this paper, we introduce a three-dimensional chaotic system by analyzing basic dynamical properties, particularly including amplitude modulation. And to study its application in multi-media security, an image encryption scheme is presented based on the permutation-diffusion architecture. The proposed cryptosystem can ensure high security by mainly employing the following two innovations: (a) The chaotic sequences for permutation and diffusion are simultaneously generated from Logistic map and new chaotic system, thus the change of one of secret keys will affect both the permutation module and the diffusion procedure. Moreover, the chaotic key stream for diffusion process is modified based on the properties of ideal pseudo-random sequence. (b) The plaintext sequence and amplitude parameter are employed to generate key stream based on the idea of OTP, which will cause a significant variation in the key stream if we choose different pixel of plain-image, different parameter or different initial condition of chaotic systems. Therefore, this procedure can effectively resist known-plaintext attack and chosen-plaintext attack. And, consequently, it can also withstand other conventional attacks. Finally, we have carried out key space analysis, key sensitivity analysis and statistical analysis to demonstrate the security of the introduced image encryption scheme.

\section{Acknowledgment}

This research was supported by the NNSF of China under Grant No. 11475060; the Research Foundation of Education Bureau of Hunan Province of China under Grant No. 16B113; the China Postdoctoral Science Foundation under Grant No. 2016M590745 and Hunan Provincial Natural Science Foundation of China under Grant 2016JJ4036.

\section{References}

[1] Zhu C. (2012). A novel image encryption scheme based on improved hyperchaotic sequences. Opt Commun, 285, 29-37.

[2] Del, R. A. M., \& Sánchez, G. R. (2015). An image encryption algorithm based on 3D cellular automata and chaotic maps. Int J Mod Phys $C$.

[3] Alvarez, G., \& Li, S. (2006). Some basic cryptographic requirements for chaos-based cryptosystems. Int J Bifur Chaos.

[4] Luo, Y., Du, M., \& Liu, J. (2015). A symmetrical image encryption scheme in wavelet and time domain. Commun Nonlinear Sci Numer Simul.

[5] Zhou, Y., Bao, L., \& Chen, C. L. P. (2014). A new 1D chaotic system for image encryption. Signal Process, $97,172-182$

[6] Wang, X. Y., Gu, S. X., \& Zhang, Y. Q. (2015). Novel image encryption algorithm based on cycle shift and chaotic system, Opt Laser Eng, 68, 126-134.

[7] Liu, S., Guo, C., \& Sheridan, J. T. (2014). A review of optical image encryption techniques. Opt Laser Technol, 57, 327-342.

[8] Muthukumar, P., Balasubramaniam, P., \& Ratnavelu, K. (2014). Synchronization and an application of a novel fractional order King Cobra chaotic system. Chaos.

[9] Yao, W., Zhang, X., Zheng, Z., \& Qiu, W. (2015). A colour image encryption algorithm using 4-pixel Feistel structure and multiple chaotic systems. Nonlinear Dyn, 81, 151-168. 
[10] Fridrich, J. (1998). Symmetric ciphers based on two-dimensional chaotic maps. Int J Bifur Chaos.

[11] Zhang, W., Wong, K., Yu, H., \& Zhu, Z. (2013).A symmetric color image encryption algorithm using the intrinsic features of bit distributions. Commun Nonlinear Sci Numer Simul.

[12] Zhang, Y., \& Xiao, D. (2014). An image encryption scheme based on rotation matrix bit-level permutation and block diffusion. Commun Nonlinear Sci Numer Simul.

[13] Lin, Z. S., Yu, S. M., Lü, J. H., Cai, S. T., \& Chen, G. R. (2014). Design and Arm-embedded implementation of a chaotic map-based real-time secure video communication system. IEEE Trans. Circuits Syst. Video Technol, 99, 1051-8215.

[14] Kong, D., \& Shen, X. (2014). Multiple-image encryption based on optical wavelet transform and multichannel fractional Fourier transform. Opt Laser Technol, 57, 343-349.

[15] Zhong, Z., Chang, J., Shan, M. G., \& Hao, B. G. (2012). Fractional fourier-domain random encoding and pixel scrambling technique for double image encryption. Opt Commun.

[16] Xiang, T., Wong, K., \& Liao, X. (2007). Selective image encryption using a spatiotemporal chaotic system. Chaos.

[17] Solak, E., Cokal, C., Yildiz, O., \& Biyikoglu T. (2010). Cryptanalysis of Fridrich’s chaotic image encryption. Int J Bifur Chaos.

[18] Rhouma, R., \& Safya, B. (2017). On the security of a spatiotemporal chaotic cryptosystem. Chaos, 17, 033117.

[19] Cokal, C., \& Solak, E. (2009). Cryptanalysis of a chaos-based image encryption algorithm. Phys Lett A, 373, 1357-1360.

[20] Zhang, Y., \& Xiao, D. (2013). Cryptanalysis of S-box-only chaotic image ciphers against chosen plaintext attack. Nonlinear Dyn, 72, 751-756.

[21] Zhu, C., Xu, S., \& Hu, Y., et al. (2014). Breaking a novel image encryption scheme based on Brownian motion and PWLCM chaotic system. Nonlinear Dyn, 79, 1511-1518.

[22] Shannon, C. E. (1948). A mathematical theory of communication. Bell Syst Tech J., 27, 379-423.

[23] Gehani, A., Labean, T., \& Reif, J. (2003). DNA-based cryptography. Lecture Notes in Computer Science, 2950, 233-249.

[24] Brandão, F., \& Oppenheim, J. (2012).Quantum one-time pad in the presence of an eavesdropper. Phys Rev Lett, 108, 040504.

[25] Nagaraj, N. (2012). One-time pad as a nonlinear dynamical system. Commun Nonlinear Sci Numer Simul, $17,4029-4036$.

[26] Yang, Q., Zhang, K., \& Chen, G. (2009). A modified generalized Lorenz-type system and its canonical form. Int J Bifur Chaos, 19, 1931-1949.

[27] Chen, G., \& Ueta, T. (1999). Yet another chaotic attractor. Int J Bifur Chaos, 9, 1465-1466.

[28] Lainscsek, C. (2012). A class of Lorenz-like systems. Chaos, 22, 013126.

[29] Lü, J., \& Chen, G. (2002). A new chaotic attractor coined. Int J Bifur Chaos, 12, 659-661.

[30] Wei, Z. (2011).Dynamical behaviors of a chaotic system with no equilibria. Phys Lett A, 376, 102-108.

[31] Li, C., Wu, L., Li, H. M., \& Tong, Y. N. (2013). A novel chaotic system and its topological horseshoe. Nonlinear Anal Model Control, 18, 66-77.

[32] Li, C., Su, K., \& Wu, L. (2013). Adaptive sliding mode control for synchronization of a fractional-order chaotic system. J Comput Nonlinear Dynam, 8, 031005.

[33] Li, C., \& Zhang, J. (2016). Synchronization of a fractional-order chaotic system using finite time input-to-state stability. Int J Syst Sci., 47(10), 2440-2448.

[34] Kerckhoffs, A. (1978). La Cryptographie Militaire. University Microfilms. 


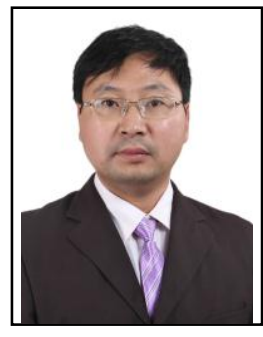

Chunlai Li received his master of engineering from the College of Electronic Engineering, Guangxi Normal University, Guilin, China in 2006. He gained his PhD from the College of Automation, Guangdong University of Technology, Guangzhou, China in 2012. He is currently a professor in the College of Physics and Electronics at Hunan Institute of Science and Technology. His current research interests include stable operation of power system, chaos control, chaos dynamics and chaos application.

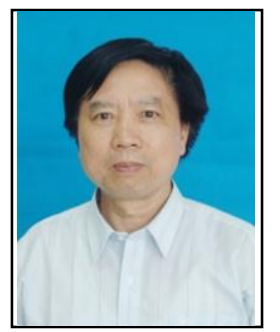

Wenhua Hai started the college physics teaching and research work from January 1982 and was promoted to professor in 1994. He was a doctoral supervisor since 2001 in Hunan Normal University. He is the author of more than 140 papers in SCI journals, including 32 papers published in the international authoritative journal Phys. Rev. His current research interests include chaos dynamics and chaos control in classical chaotic system, the atomic and molecular system and the condensed matter. 